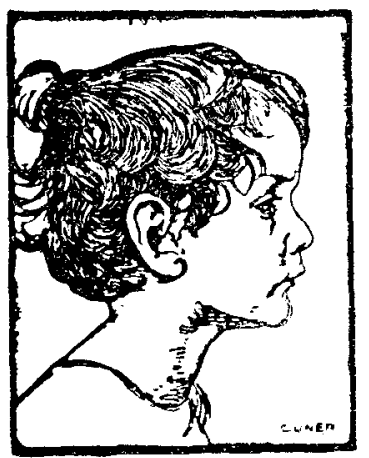

\title{
"Pro Juventute”
}

Revue mensuelle illustréc pour la protection de la jeunesse.

Cette revue, qui publie des articles en français, allemand et italien et est dirigée dans un esprit de parfaite neutralité religieuse et politique, se propose de devenir de plus en plus le centre de ralliement de toutes les cuvres pour la protection de la jeunesse en Suisse et de nouer des relations suivies avec les cuvres similaires de l'étranger.

Sa lecture présente donc un intérêt très réel pour toutes les personnes qui comprennent l'importance primordiale qu'offre à notre époque la formation d'une jeunesse forte et saine.

Prix de l'abonnement : frs. 7.- par an.

On s'abonne à tous les bureaux de poste suisses ainsi quau Secrétariat central Pro Juventute, Untere Zäune I I, Zurich, I. Pour les annonces, s'adresser au Secrétariat central.

Compte de chèques postaux, VIII 3 roo, Zurich.

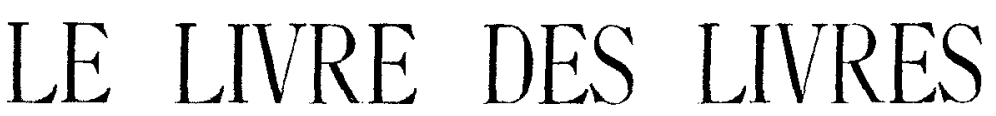

Nous sommes heureux de signaler, à ceux de nos lecteurs qui ne la connaîtraient pas, cette anthologie critique mensuelle des nouveaux ouvrages littéraires, dont chaque numéro, qui contient la Critique, l'Analyse d'importants Extrails (texte et illustrations) des volumes récemment parus, permet I $^{\circ}$ d'être rapidement et bien au courant des dernières productions; $2^{\circ}$ de fairc son choix en connaissance de cause.

Une telle revue cst particulièrement utile à notre époque ou les livres coûtent si cher!

Abonnement : France, un an, $14 \mathrm{fr}$; six mois, $7 \mathrm{fr} .50$; trois mois, $4 \mathrm{fr}$. - Etranger, un an, $16 \mathrm{fr}$; six mois, $8 \mathrm{fr} .50$; trois mois, 4 fr. 50 . - Le numéro: France, 1 fr. 50 ; Etranger, 1 fr. 70.

Une augmentation de prix est à craindre.

Il reste quelques rares collections des $\mathrm{I} 2$ premiers numéros parus, qui sont expédiés en France, contre $\mathrm{I}_{4}$ fr. et à l'étranger contre $\mathrm{I} 6 \mathrm{fr}$.

Adresser la correspondance au directeur, M. Gaston Moussé, 3, rue du Marché des Patriarches, Paris ( $\left.V^{m e}\right)$. 


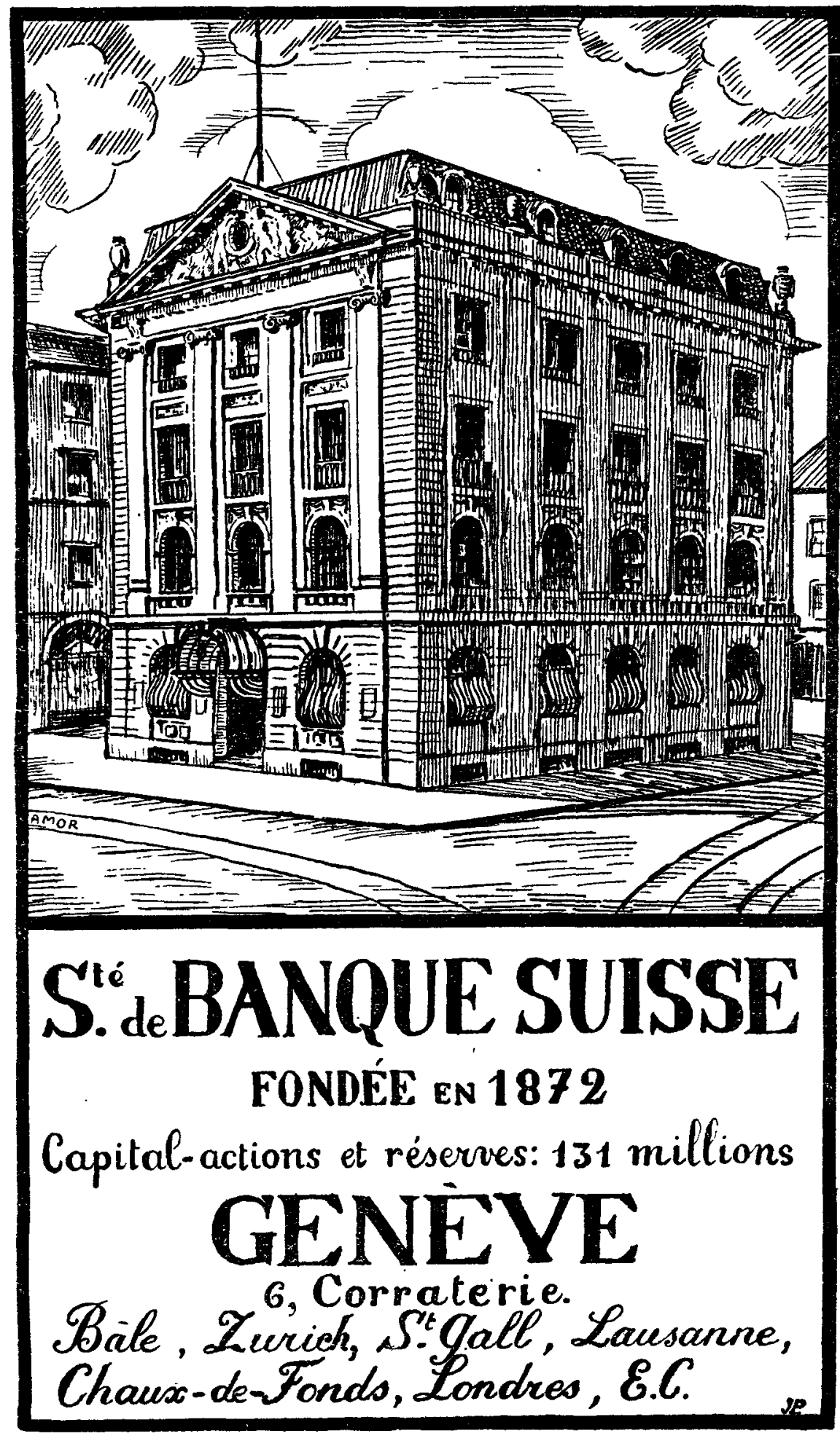




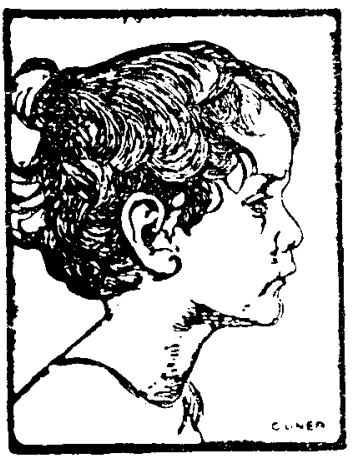

"Pro Juventute”

Revue mensuelle illustrée

pour la protection de la jeunesse.

Cette revue, qui publie des articles en français, allemand et italien et est dirigée dans un esprit de parfaite neutralité religieuse et politique, se propose de devenir de plus en plus le centre de ralliement de toutes les cuvres pour la protection de la jeunesse en Suisse et de nouer des relations suivies avec les œuvres similaires de l'étranger.

Sa lecture présente donc un intérêt très réel pour toutes les personnes qui comprennent l'importance primordiale qu'offre à notre époque la formation d'une jeunesse forte et saine.

Prix de l'abomnement : frs. 7.- par an.

On s'abonne à tous les bureaux de poste suisses ainsi qu'au Secrétariat central Pro Juventute, Untere Zäune II, Zurich, I.

Pour les annonces, s'adresser au Secrétariat central.

Compte de chèques postaux, VIII 3 roo, Zurich.

\section{On demande des dames ou jeunes}

filles volontaires pour aider aux distributions de secours en Pologne, tous frais payés. - - S'adresser au British Committee for Relief in Poland, Miss G. C. Vulliamy, C. B. E., Senatorska, 35, Varsovie. 


\title{
VIENT DE PARAITRE : \\ L'Union internationale de Secours aux Enfants
}

\author{
Genève, 4, rue Massot, r920. \\ In-8, 48 et roo $p$.
}

Album contenant les portraits de membres du Comité d'honneur de l'Union, les autographes de huit membres du Comité d'honneur, les listes des Comités collecteurs affiliés à l'Union en Allemagne, Arménie, Finlande, France, Grande-Bretagne, Italie, Pays-Bas, Suède, Suisse, Turquie et 75 photographies de groupes d'enfants éprouvés des pays suivants : Albanic, Allemagne, Albanie, Autriche, Belgique, Esthonie, France, Hongrie, Italie, Lettonie, Pologne, Roumanie, Russie, Serbie (S. H. S.), Turquic.

Prix : 5 frs. (argent suisse).

\section{BULLETIN DE L'UNION INTERNATIO- NALE DE SECOURS AUX ENFANTS}

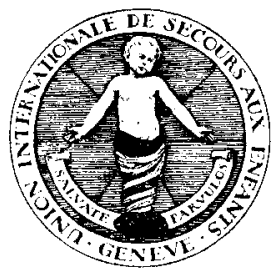

Save the Children Fund: International Union Internationale Vereinigung für Kinderhilfe Unione Internationale per la salvezza dei bambini

sous le patronage du

COMTÉ INTERNATIONAI. DE LA CROIX-ROGGH

Rédaction et Administration :

4, Rue Massot, Genève (Suisse)

Paraît sur 12 ou 16 pages in-8, les ro, 20 et 30 de chaque mois. A la plupart des numéros est adjoint un supplément illustré de 4 pages en photogravure. Prix de l'abonnement 9 frs. par an. Sous la forme la plus concise ce bulletin trimestricl relate les faits les plus marquants du mouvement international du secours aux enfants des pays éprouvés par la guerre, collectes catholiques, protestantes, orthodoxes, israélites, collectes socialistes, etc., allocations aux cuvres de secours aux enfants, rapports sur l'activité de ces œuvres tant américaines qu'européennes, renseignements impartiaux sur la misère des enfants, en Europe et dans le Proche Orient.

L'Union internationale de secours aux enfants n'a aucun caractère politique ni confessionnel, elle se borne à recueillir et à répartir des fonds et s'abstient de toute action directe. 


\section{LA REVUE DE GENÈVE}

PARATT TOUS LES MOIS, SUR

I60 PAGES IN-8 $8^{\circ}$ AU MINIMUM

Internationale, mais non internationaliste, intersociale mais non socialiste, la Revue de Genève est une revue de liaison intellectuelle et de documentation originale. Seule, elle joue ce rôle nécessaire à une époque qui, après tant de bouleversements, réclame qu'on reconstruise. Dans ce chaos douloureux, elle cherche à retrouver les lignes directrices; elle contribue à préserver notre civilisation soumise à tant de menaces.

Chacun de ses numéros se divise en trois rubriques. Une première partie contient des ouvres d'imagination, des études de caractère général, des essais politiques, historiques, critiques. La seconde partie donne des "Chroniques nationales", lesquelles sont rédigées par des ressortissants des pays dont elles traitent. Tirant la philosophie des événements, ces chroniques fournissent des vues d'ensemble et donnent un tableau comparé, une image synthétique du monde moderne.

A ces "chroniques nationales" succède une "chronique internationale " consacrée à retracer les efforts des peuples non plus pour s'affirmer, mais pour s'entendre les uns les autres. On y trouve l'analyse des grands problèmes qui se posent à toutes les nations en commun, la libre discussion des diverses institutions universelles dont Genève est le siège, le compte rendu de l'activité internationale dans le monde entier.

La Revue de Genève compte parmi ses collaborateurs : MM. Mauric: Farrès, René Boylesve, Georges Duhamel, Edouard Estaunié, Georges Eekhoud, Elie Faure, Daniel Halévy, Emile Henriot, Edmond Jaloux, Camille Mauclair, Pierre Mille, Edmond Pilon, Tenri de Régnier, Jean Richard-Bloch, Jules Romains, André Suarès, J. J. Tharaud, Albert Thibaudet, Jean-Louis Vaudoyer, Benedetto Croce, G. Ferrero, Piero Jahier, G. Papini, Vilfredo Pareto, G. Prezzolini, Joseph Conrad, Georges Moore, Bernard Shaw, J. Zangwill, Arnold Bennett, John Erskine, Charles Macfarland, E. Curtius, F. W. Förster, Freud, H. Kessler, Heinrich Mann, W. Rathenau, J. Redlich, Maxime Gorki, Kouprine, Remisov, Sologoub, J. Bojer, Geijerstmamm, Per Hellstroem, Jules Andrassy, Fr. Riedl, J. de Voïnovitch, Andreades, N. Jorga, Ad. Salazar, Alfonso Reyes, Miguel Unamuno, Graça Aranha, etc., ainsi que les écrivains suisses les plus importants.

On s'abonne à la Revue de Genève, 46, rue du Stand, Genève (Editions Sonor), et chez les principaux libraires.

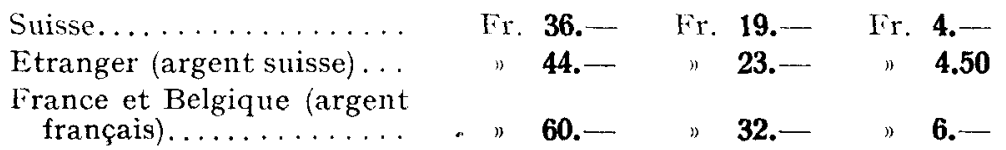




\section{JOURNAL OF COMPARATIVE LEGISLATION}

and

\section{INTERNATIONAL LAW}

October 1920.

Six Shillings.

Industrial Courts in Australia : By the Hon. Mr. President Jethro Brown, Industrial Court of South Australia.

United States Senate and the Treaty-making Powers : By Donald McMaster, K. C., D. C. L., M. P.

The Imperial Crown and the Foreign Relaitons of the Dominions :

By H. Duncan Hall, M. A., B. Litt.

A New Basis for Industrial Corporations : By G. Stirling Taylor. Land Settlement of Ex-Service Men in Australia, Canada and the United States. By A. J. Hannan, Parliamentary Draftsman, Sonth Australia.

The Noutrality of the Channel Islands during the 15th, I6th, and Centuries, : By E. Toulmin Nicolle, Viscomte of Jersey.

Some Recent Writers on Criminal Law. By Professor Courtney Ke. ney.

State Immunity in the Laws of England, France, Italy and Belgimm By Professor F. P. Walton, LL. D.

Mining Laws of the West African Colonies and Protectorates. By Gilbert Stone.

The New Constitution of Peru : By Wyndham A. Bewes.

The Alien Enemy in English Law : By R. F. Roxburgh.

Private Law in China. By J. E. G. de Montmorency.

Ships and their Owners in the Prize Courts of France, Crt. Britain, Italy and China. By C. J. Colombos.

Justice and the Poor. By Hon. Alderson Smithson.

Merchandise Marks Committee : By Hon. H. Flecther Moulton, The Freedom of the Seas : By. G. G. Phillimore.

The Theory of Mohammedan Law: By Ahmed Safwat, public Prosecutor, Cairo.

Wills of Lunatics : By W. G. H. Cook, D. D.

Notes on Points of Imperial Constitutional Law. By Prof. Berriedale Keth.

International Bar Association.

Notes. Notices of Books.

Annual Subscription, One Guinea.

Society of Comparative Legislation.

1, Elm Court, Temple; London E. C. t, England 
CUBA. - Société nationale cubaine de la Croix-Rouge, rue O'Reilly, 6 La Havane.

DANEMARK. - Société danoise de la Croix-Rouge, Ved Stranden, 2, Co penhague, $\mathrm{K}$.

ESPAGNE. - Société espagnole de la Croix-Rouge, Atocha, 65, Madrid.

ÉTATS-UNIS. - Central Committee of the American National Red Cross Washington, D. C.

FINLANDE. - Direction centrale de la Croix-Rouge de Finlande, Helsingfors

FRANCE. - Comité central de la Croix-Rouge française, rue François Ies 21, Paris, $8^{\mathrm{m} \varepsilon}$.

GRANDE-BRETAGNE. - British Red Cross Society, 19, Berkeley Street Londres, IV. I.

GRÉCE. - Croix-Rouge hellénique, Athènes.

HESSE. - Société hessoise de la Croix-Rouge, Darmstadt.

HONGRIE. - Société hongroise de la Croix-Rouge, Andrassy-ut, 8, Budapesl, VI.

ITALIE. - Croix-lRouge italienne, via Toscana, 10, Rome.

JAPON. - Société japonaise de la Croix-Rouge, Tokio.

LUXEMBOURG. - Société luxembourgeoise"de la Croix-Rouge, Luxembourg

MEXIQUE. - Comité central de la Société mexicaine de la Croix-Rouge 2 a de San Geronimo, 14, Mexico, D. F.

MONTÉNÉGRO. - Comilé central de la Croix-Rouge monténégrine, ru Ancelle, 9, Neuilly-sur-Seine.

NORVĖGE. - Socicté norvégienne de la Croix-Rouge, Akersgaten, 44 Christiania.

PAYS-BAS. - Comité Supérieur de la Croix-Rouge néerlandaise, Princes segracht, 29, La Haye.

PÉROU. - Société péruvienne de la Croix-Rouge, Lima.

POLOGNE. - Société polonaise de la Croix-Rouge, Mazowiecka, 9, Varsovit

PORTUGAL. - Société portugaise de la Croix-Rouge, Lisbonne.

PRUSSE. - Comité central de l'Association prussienne de la Croix-Rouge am Karlsbad, 23, Berlin, W. 35 .

ROUMANIE. - Société nationale de la Croix-Rouge de Roumanie, Acade

RUSSIE. miei, 20, Bucarest.

SAXE. - Société saxonne de la Croix-Rouge, Dresde.

SERBIE. - Socièté serbe de la Croix-Rouge, Simina ulica, 21, Belgrade.

SIAM. - Société siamoise de la Croix-Rouge, Bangkok.

SUĖDE. - Comité central de la Société suédoise de la Croix-Rouge, Artille rigatan, 6, Stockholm, 5 .

SUISSE. - Croix-Rouge suisse, rue des Cygnes, 9, Berne.

TCHÉCOSLOVAQUIE. - Société tchécoslovaque de la Croix-Rouge, Pragut

TURQUIE. - Comité central de la Société du Croissant-Rouge ottoman Mahmoudié Djaddessi, Constantinople.

URUGUAY. - Comité central de la Croix-Rouge uruguayenne, calle Colon 1382, Montevideo.

VENEZUELA. - Senor Presidente de la Sociedad Venezolana de la Cruz-Roja Este 6, No 55, Caracas.

WURTEMBERG. - Société wurtembergeoise de la Croix-Rouge, Stuttgart. 


\title{
Sommaire du Bulletin
}

\author{
$N \cdot 221$
}

Comité international. - Circulaires : Distribution des revenus du

Fonds de l'impératrice Shôken ( $\mathrm{n}^{\circ} 20 \mathrm{I}$ ), 29. - Décès de M. Alfred Gautier ( $\mathrm{n}^{\circ} 202$ ) (avec portrait), 3o. - Préparation de la $\mathrm{X}^{\mathrm{me}}$ Conférence internationale des Croix-Rouges, 33. - Attribution d'une médaille Florence Nightingale à la Croix-Rouge australienne, 35 . - Situation du Fonds Augusta au 7 janvier I921, 36. - Situation du Fonds Nightingale, 36. - Situation du Fonds de l'impératrice Sbôken, 37. - Protestation, 37. - Rapatriement des mineurs grecs et libération des prisonniers bulgares, 38. - Les réfugiés russes, 39. - Action du Comité international en faveur des enfants de Budapest, 46. - Missions et délégations, 47. - Personnel des Comités centraux, 49 .

Ligue. - Un article du Bulletin de la Ligue, 52.

Belgique. - Nouveaux statuts de la Croix-Rouge belge du ler $^{\text {er }}$ septembre 1920,53 .

Espagne. - L'école d'infirmières rétribuées de la Croix-Rouge espagnole, 62 .

Esthonie. - Détresse des enfants, 64 .

France. - La Société de secours aux blessés militaires dans le proche Orient, 65.

Grande-Bretagne. - Protection du signe de la Croix-Rouge, 66.

Grèce. - Sections étrangères sur territoire national, 66 .

Italie. - Les secours de la Croix-Rouge internationale aux sinistrés du tremblement de terre dans les régions de la Lunigiana et Carfagnana (avec carte), $6 \mathrm{~g}$.

Lettonie. - Détresse des enfants, 74 .

Norvège. - Comité pour la lutte contre la famine, 75 .

Pologne. - Activité de la Croix-Rouge polonaise en juillet-août r $9^{20}$, 76.

Siam. - Loi sur la Croix-Rouge du Siam et statuts du 2 I octobre 1920, 77 .

Tchẻcoslovaquie. - Rapatriement des prisonniers et réfugiés (avec carte), 83.

Ukraine. - Activité de la Croix-Rouge ukrainienne, 86.

La Revue Internationale de la Croix-Rouge, Bulletin International des Sociétés de la Croix-Rouge paraît le i 5 de chaque mois.

Prix, franco, un an : Fr. 20.- Le numéro: Fr. 2.-

Pour l'Espagne et l'Amérique latine: 25 pesetas

Année 1919 (rare) : Fr. 30.-

Administration : Promenade du Pin, 1, Genève.

Pour la publicité de la Revue, s'adresser à Orell Fussli-Publicité, rue de la Croix-d'Or, 2-4, Genève, et à ses succursales: Lausanne, Neuchâtel, Barne, Bâle, Soleure, Aarau, St-Gall, Zurich. 